\title{
Wirkungen erforschen und dokumentieren
}

\section{Die Kinder- und Jugendarbeit muss ihre Mess- und Erhebungsinstrumente schärfen}

\author{
Werner Lindner
}

\begin{abstract}
Die Forderung nach »Evidenzbasierung" erreicht die Kinder- und Jugendarbeit. Finanzierungen werden immer öfter an den Nachweis von " Wirkungen " gekoppelt. Bei aller Vorsicht gegenüber tagespolitischen Ansprüchen weiß die Soziale Arbeit gleichwohl noch wenig über ihre Erfolgsbedingungen, über die Frage der Übertragbarkeit von Ergebnissen und über die Frage, mit welchen Methoden welche Wirkungen erzielt werden können.
\end{abstract}

In der Sozialen Arbeit ist Evaluation nichts Neues. Das Thema scheint aber unter den Vorzeichen der »Evidenzbasierung " an Bedeutung zu gewinnen (vgl. aktuell Sommerfeld/Hüttemann 2007), wobei bislang ein theoretisch-programmatischer Überschuss zu verzeichnen ist, hinter dem die Praxis einstweilen zurück bleibt.

Dieser Bedeutungsgewinn läuft darauf hinaus, die Praxis der Sozialen Arbeit zusehends auf die Erhöhung der bemessbaren Wirksamkeit ihres Outputs zu verpflichten. Nicht mehr Wirkungsversprechen, nicht mehr Struktur- oder Prozessqualität, sondern Wirkungsgarantien und Ergebnisqualität zählen und aktualisieren die Formel »money for value «, derzufolge sich künftig Finanzierungen an das Vorliegen von Wirksamkeitserfolge koppeln.

$\mathrm{Ob}$ und inwiefern sich dieser Trend langfristig durchsetzt, bleibt mindestens abzuwarten, gewiss aber sorgfältig zu reflektieren. In der Kinder- und Jugendarbeit sind in den letzten Jahren etliche Evaluationsvorhaben durchgeführt worden (Lindner 2008), die geeignet sind, bislang bestehende »Fenster der Verwundbar-

\footnotetext{
Prof. Dr. Werner Lindner ist Hochschullehrer für Jugendarbeit, jugendliche Ausländer, Jugendkultur an der Fachhochschule Jena.

E-Mail Werner.Lindner@fh-jena.de
}

keit « zu schließen und die erweisen, dass sich dieses Arbeitsfeld weniger auf Programmformeln (z. B. Bildung) oder pädagogisch wohlmeinende Absichten (z. B. Prävention) stützt, sondern zusehends auf valide Daten und Fakten. In ihren KernBereichen (politische Bildung, jugendkulturelle Bildung, internationale Jugendarbeit, verbandliche und offene Jugendarbeit) kann die Kinder- und Jugendarbeit eine Vielzahl differenziertester Bildungseffekte und Kompetenzzuwächse wie auch Langzeitwirkungen nachweisen. Diese Feststellungen verbinden sich mit weiteren Erkenntnissen, Erfahrungen und Hinweisen für eine künftig noch optimiertere Praxis der Zielerreichung.

Zugleich sind eine Reihe von kritischen Punkten zu markieren, die vor einem prompten Aufspringen auf einen womöglich verheißungsvollen Zug eher warnen sollen. Gerade die Kinder- und Jugendarbeit hatte sich in den letzten Jahren verschärften Legitimationsanforderungen zu stellen. Vor dem Hintergrund vielfach irrigen fachöffentlichen Einschätzung als allenfalls "weiche « Aufgabe des Kinderund Jugendhilfegesetzes (SGB VIII) sind hier insbesondere in den neuen Bundesländern gravierende Mittel- und Personalkürzungen erfolgt. So ist auf der einen Seite zu fragen, wie sich die Anforderungen nach Wirkungen umsetzen lassen sollen angesichts parallel erfolgter Verunmöglichung angemessener Arbeits- und Rahmenbedingungen durch permanentes Drehen an den Finanzierungsschrauben. Selbstverständlich gilt auch für die Kinder- und Jugendarbeit, »dass Evaluation darauf abzielt, durch eine datenbasierte Bewertung von Handlungsalternativen zu einer rationaleren Entscheidungsfindung beizutragen « (Heiner 2001, S. 491). Zugleich aber ist in Rechnung zu stellen, dass »Evaluationsmodelle (....) ebenso wie Evaluationen auf Wertentscheidungen beruhen, die nicht hinreichend durch wissenschaftliche Analysen begründet werden können. So lässt sich die Auswahl von Evaluationskriterien zwar wissenschaftlich untersuchen (Welcher Logik folgen sie? Wen begünstigen sie? Was wird auf diese Weise ausgeblendet?). Sie lassen sich damit aber nicht zwingend begründen. " (Heiner, ebd.)

Die fraglos heikle, aber wohl unvermeidbare Kopplung von Evaluationsergebnissen an Nutzerorientierungen ist mithin sorgfältig zu beachten. Dies gilt insbesondere dort, wo zwar gern »innovative " Lösungen oder Wirkungen erwartet (oder explizit gefordert) werden, diese aber innerhalb eines politisch oder administrativ zumeist restriktiven und vorab gesetzten Rahmens zu erfolgen haben.

Gerade hier steht die Frage zu Beantwortung an: »(Wer) definiert wie zu welchem Zeitpunkt den jeweiligen Nutzen, und wie kann für eine Evaluation entschieden werden, was jeweils wann als nützlich zu gelten hat? « (Haubrich/ Lüders/ Struhkamp 2007; S. 193) Evaluation lässt sich also nicht auf pure Forschung reduzieren, denn sie wird als Beratungsinstrument für die Politik (auf kommunaler, Landes- oder Bundesebene) eingesetzt, die über die Gewährung und Legitimation von Ressourcen entscheidet; sie ist ein "Balanceakt zwischen Wissenschaft und Politik « (Bewyl 1988 zitiert nach Heiner 2001, S. 482). Ohne das Bedenken des Entstehungs- und Verwertungszusammenhangs und ohne den Bezug auf fachliche Debatten sowie gesellschaftspolitische Rahmenbedingungen, gerät Evaluation zu beliebig-funktionaler Evidenztechnik.

Wirkungen der Kinder- und Jugendarbeit werden von der Politik vorzugsweise dann angefragt, wenn Legitimationen benötigt werden oder die Umverteilung finanzieller Mitteln ansteht, was oftmals miteinander verwandt ist. Als etwa im August 2006 Kürzungen in der sächsischen Jugendverbandsarbeit verkündet wurden, räumte der zuständige Staatssekretär zwar ein, dass sich die Arbeit der 
überörtlichen Jugendverbände "grundsätzlich bewährt « habe; gleichwohl sei dem gesellschaftlichen und demografischen Wandel Rechnung zu tragen mit der Konsequenz für die Verbände, »die Effizienz und Wirksamkeit ihrer Arbeit zu beleuchten « (http://www.jugendhilfeportal.de, Mitteilungen vom 23.8. 2006).

Ob Evaluationen hier die Abhilfe schaffen, darf bezweifelt werden. Denn das Beispiel etwa des Wirksamkeitsdialoges in Nordrhein-Westfalen erweist, dass auch die ambitioniertesten Modelle ausbluten, wenn es die Politik nicht mehr interessiert und »unvermeidliche" Einsparungen anstehen. Allein die hierbei sich aufdrängende (hypothetische) Frage, ob die Kürzungen etwa zurückgenommen würden, wenn die Ergebnisse entsprechend positiv ausfielen, mutet naiv an. Vielmehr ist darauf hinzuweisen, dass solche Kürzungen sich weder fachlich, noch rechtlich noch finanziell noch bildungs- oder entwicklungsstrukturell als » unvermeidlicher Sachzwang « oder sonst wie plausibel legitimieren lassen, sondern höchstens mit der weitgehenden Abwesenheit einer verantwortbaren Jugendpolitik, die von einem Aktionsprogramm bis zum nächsten denkt und sich im Zweifelsfall von Wirkungsfakten nicht im geringsten beeindrucken lässt, wenn es ihr darum geht, Stimmung zu machen (vgl. Wahlkampf 2008 in Hessen und Bayern). Fazit: Evaluationen schützen vor gar nichts - trotzdem müssen sie geleistet werden; mindestens aus Gründen eigener Professionalitätsansprüche.

Es ist weiterhin - um im Sprachgebrauch zu bleiben - evident, dass hier eine ganze Reihe altbekannter Probleme im neuen Gewande auftauchen, als da sind - die Frage der Anwendung wissenschaftlichen Wissens durch Praktikerinnen und Praktiker (Dewe/Ferchhoff/ Radtke 1992)

- das Verhältnis von Wissen und Tun (Klatezki 1993)

- die Option für Praktiker, sich auch als Forscher in eigener Sache zu entwickeln (Müller 2001, Evangelische Akademie 2000).

$\mathrm{Zu}$ vergegenwärtigen ist schließlich, dass mit dem Evidenzanspruch gerade in der Kinder- und Jugendarbeit - einstweilen - zwei Welten aufeinandertreffen: Evidenzbasierung beruht auf empirischen Ergebnissen, die sich am Optimum des sogenannten »Goldstandards «, nämlich
Meta-Analysen im Vergleich der Mittelwerte von randomisierten Kontrollstudien orientieren. Nun ist aber die allgemeine Forschungslage gerade in der Kinder- und Jugendarbeit, trotz mancher Verbesserungen, immer noch ausgesprochen schwach. Brauchbare Datenbanken und Meta-Analysen sind einstweilen $\mathrm{Zu}$ kunftsmusik, so dass Praktikerinnen und Praktiker, selbst wenn sie evidenzbasiert arbeiten wollten, allenfalls in ein Evaluationsvakuum vorstießen. Gerade in der gefallen lassen muss. Und solange manche Praxisfelder zu alledem blind für ihr eigenes Nichtwissen sind, wird man dem auch mit Evidenzbasierung kaum abhelfen können.

In dem Maße, wie sich Evidenzbasierung als reale, fachlich ertragreiche Professionalisierungsperspektive fundierte, wäre die Kinder- und Jugendarbeit selbstverständlich herausgefordert, auf breiter Front Anschluss zu gewinnen an die Etablierung einer evidenzbasierten Praxis

\section{»Das Erreichen von Wirkungen setzt angemessene Arbeits- und Rabmenbedingungen voraus «}

Praxis der Kinder- und Jugendarbeit sind die Voraussetzungen für die Beantwortung von Wirkungsfragen dünn aufgrund einer nach wie vor unzureichenden Forschungskultur, die sich bis auf weiteres auch den Vorwurf der Empirieabstinenz und dabei - im Blick auf die bisherigen Ergebnisse - die Erfolge der eigenen Arbeit weder gering zu schätzen, noch in Selbstgefälligkeit und Größenwahn zu verfallen. Vielmehr wären ihre Erkenntniszugewinne mit aller Konzentration

\section{Literatur}

Dewe, B./Ferchhoff, W./Radtke, F.-O. (1992): Das »Professionswissen « von Pädagogen. Ein wissenstheoretischer Rekonstruktionsversuch. In: (Dies.) (Hg.): Erziehen als Profession. Zur Logik professionellen Handelns in pädagogischen Feldern. Opladen, S. 71-92.

Evangelische Akademie Loccum (2000): Mit der Jugendforschung zur besseren Praxis? Oder: Welche Forschung braucht die Jugendarbeit? Loccumer Protokoll 63/00; herausgegeben von A. Grimm. Rehburg-Loccum.

Haubrich, K./Lüders, Ch./Struhkamp, G. (2007): Wirksamkeit, Nützlichkeit, Nachhaltigkeit. Was Evaluationen von Modellprogrammen realistischerweise leisten können. In: Schröder, U./Streblow, C. (2007) (Hg.): Evaluation konkret. Fremd- und Selbstevaluationsansätze anhand von Beispielen aus Jugendarbeit und Schule. Opladen, Farmington Hills, S. 183-201.

Heiner, M. (2001): Evaluation. In: Otto, H.-U./Thiersch, H. (Hg.): Handbuch Sozialarbeit/Sozialpädagogik. 2. überarbeitete Auflage. Neuwied, S. 481-495.

Klatezki, Th. (1993): Wissen, was man tut. Professionalität als organisationskulturelles System. Eine ethnographische Rekonstruktion. Bielefeld.

Lindner, W. (2008) (Hg.): Kinder- und Jugendarbeit wirkt. Aktuelle und ausgewählte Evaluationsergebnisse der Kinder- und Jugendarbeit. Wiesbaden.

Sommerfeld, P./Hüttemann, M. (2007) (Hg.): Evidenzbasierte Soziale Arbeit. Nutzung von Forschung in der Praxis. Baltmannsweiler.

Müller, B. (2001): Praktiker als Forscher - forschen als Praxis: Eine Wahlverwandtschaft. In: neue praxis 1/2001, S. 3-8.

Schrödter, M./Ziegler, H. (2007): Was wirkt in der Kinder- und Jugendhilfe? Internationaler Überblick und Entwurf eines Indikatorensystems von Verwirklichungschancen. Wirkungsorientierte Jugendhilfe. Band 02. Münster. 
weiter auszubauen und zu fundieren und sich somit an eine auf Wirksamkeit ausgerichtete Praxis heranzutasten. Dann allerdings besteht Optimierungsbedarf in den nachfolgenden Punkten.

Bislang erfolgten die entscheidenden Evaluationsanstöße selten intrinsisch, sondern extrinsisch (durch Politik) und erzeugten notorisch ein Gefühl des Ertappt-Werdens, der Verlegenheit und des schlechten Gewissens. Hier ist die Kinderund Jugendarbeit gefragt, künftig stärker selbst die Initiative zu ergreifen, sich nicht unter Verweis auf die Ergebnisse einzelner Leuchtturm-Projekte auszuruhen, sondern diese auf der lokalen und regionalen Ebene weiter voranzutreiben. Denn Wirkungsforschung ist vor allem dann fruchtbar, "wenn sie keine leitlinienförmige Praxisanleitung, sondern ein empirisch fundiertes Arsenal an Deutungs-, Erklärungs- und damit Reflexionswissen bereitstellt, das eine Basis für eine angemessene - und d. h. vor allem dem Einzelfall angemessene - sozialpädagogische Praxis bereitstellen kann. " (Schrödter/ Ziegler 2007, S. 43)

Wo die Übergänge von Evaluationen gegenüber verwandten, aber keineswegs identischen Instrumenten wie Praxisberichten, angeleiteten Reflexionen, Praxisberatungen, wissenschaftlicher Begleitung, Prozessbegleitung, »Monitoring " oder »Qualitätsentwicklung « nicht eindeutig sind, wird bereits die Qualität der Ausgangsfragestellungen und der damit verbundenen Datenerhebungsinstrumente in Mitleidenschaft gezogen - von der Beachtung und Einhaltung solch anspruchsvoller Güte- und Qualitätskriterien gar wie des Handbuches der Evaluationsstandards noch zu schweigen. Hier wäre schon viel gewonnen, wenn die Kinder- und Jugendarbeit anfinge, im je eigenen Aktionskreis daten- und faktenbasiert zu arbeiten, um auf solcher Grundlage die Arbeit zu dokumentieren.

Die Kinder- und Jugendarbeit ist gefordert, ihre Mess- und Erhebungsinstrumente zu schärfen. Dies gilt etwa für die Verständigung über Erfolgsbedingungen und Vergleichskriterien von Indikatoren und Wirkungen, für die Frage der Übertragbarkeit von Ergebnissen, aber auch für vergleichende Wirkungszusammenhänge und der Frage, mit welchen Methoden welche Wirkungen erzielt werden.
Zudem besteht Bedarf an alltags- und praxistauglichen Prüfinstrumenten und Indikatoren und an vergleichbaren Qualitätsdimensionen (für die hier bislang kaum Voraussetzungen und operationalisierte Grundlagen vorhanden sind). $\mathrm{Zu}$ entwickeln und zu befördern wären die Erarbeitung begründeter Hypothesen über die Funktionsweise des jeweiligen Arbeitsfeldes, die Entwicklung von Arbeitshilfen, Fort- und Weiterbildungen und die Verbindung von Evaluationser- gebnissen mit der Jugendhilfe- und Sozialplanung.

Vor diesem Hintergrund und den jetzt schon vorliegenden Ergebnissen wäre jedenfalls neu einzutreten in die öffentliche Auseinandersetzung um den Stellenwert und die Perspektiven von Kinder- und Jugendarbeit, in das breit angelegte Umschalten von Qualität auf Qualität, in eine auf Dauer gestellte praxisintegrierte Forschung sowie in eine neue, empirisch fundierte jugendpolitische Offensive.

\section{Unverzichtbare Informationsquelle}

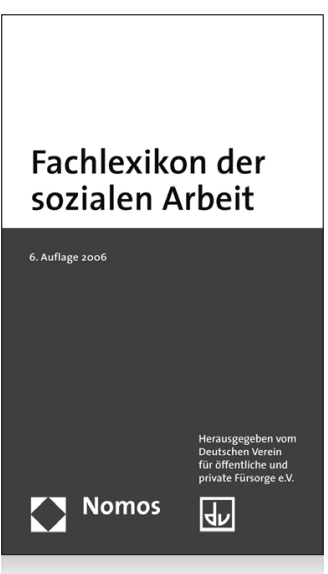

\author{
Fachlexikon der sozialen Arbeit \\ Herausgegeben vom Deutschen \\ Verein für öffentliche und private \\ Fürsorge e.V. \\ 6. Auflage 2007, 1.195 S., brosch., \\ 44,- $€$ (Vorzugspreis für Mitglieder \\ des Deutschen Vereins und Studenten \\ $34,-€)$, ISBN 978-3-8329-1825-5
}

Wesentliche Veränderungen in Gesetzgebung und Rechtsprechung haben zu einer gründlichen und umfangreichen Überarbeitung des Fachlexikons geführt.

Die Neuauflage des in der Praxis bestens eingeführten Lexikons umfasst über 1.600 Stichwörter - zusammengestellt von 673 Expertinnen und Experten. Sorgfältig eingesetzte Querverweise bieten rasche Orientierung. Das ausführliche Literaturverzeichnis erschließt mit mehr als 4.00o Titeln zusätzliche Informationsquellen.

Bitte bestellen Sie bei Ihrer Buchhandlung oder bei Nomos | Telefon 07221/2104-37| Fax -43| www.nomos.de | sabine.horn@nomos.de 